\title{
On the dependence of topographically forced flows on the form of dissipation
}

\author{
by \\ Hirotada Kanehisa \\ Meteorological Research Institute, Tsukuba, Ibaraki, 305 Japan
}

(Received November 17, 1992 ; Revised October 5, 1993)

\begin{abstract}
The dependence of topographically forced flows in a quasigeostrophic 2-layer model on the form of dissipation is investigated. In particular, the following 2 cases are examined. (1) The dissipation is proportional to the potential vorticity, and (2) the dissipation is proportional to the Laplacian of the streamfunction, i.e., Ekman dampings on the upper and lower boundaries.

In the case (1), the bifurcation diagram is qualitatively the same as Pedlosky (1981)'s in which the dissipation is a sum of Ekman dampings on the upper and lower boundaries and an interfacial friction between the upper and lower layers. That is, there exist 2 steady solution bifurcation points, between which 2 stable solutions and 1 unstable one coexist for the same parameter values of the basic zonal flow.

On the other hand, in the case (2), a wave-wave interaction occurs. As a result, although the steady solution diagram is not altered qualitatively, Hopf bifurcation points may emerge on the stable steady solution curve, and the steady solutions between them may lose their stability.

So far as the considerations in this note are concerned, the stability of steady solutions is dependent on the form of dissipation although the steady solution diagram itself is not altered qualitatively.
\end{abstract}

\section{Introduction}

Since Phillips (1954), the evolution of baroclinic waves has been investigated by means of a quasigeostrophic 2-layer model. In the model, the parameterization of dissipation due to smaller scale waves has some ambiguities.

Pedlosky (1970, 1971, 1972) investigated the nonlinear evolution of linearly unstable baroclinic waves in the quasigeostrophic 2-layer model, and obtained the following results. If the magnitude of dissipation is sufficiently large, then the wave approaches a

(C) 1993 by the Meteorological Research Institute steady state. On the contrary, if the magnitude is sufficiently small, then the wave approaches a cyclic state. Moreover, Pedlosky and Frenzen (1980) showed that the wave does not approach a steady or a cyclic state, but behaves chaotically for some intermediate value of dissipation. Thus, the evolution of baroclinic waves has been shown to depend crucially upon the magnitude of dissipation.

In the above-mentioned investigations, the dissipation was assumed to be proportional to the Laplacian of the streamfunction, i.e., Ekman damping. Other forms of dissipation were not examined.

However, using a weakly nonlinear technique, Klein and Pedlosky (1992) revealed 
that the wave approaches a steady state for any value of magnitude of dissipation if dissipation is taken to be proportional to the potential vorticity. That is, the evolution of baroclinic waves has been shown to depend crucially upon the form as well as the magnitude of dissipation.

On the other hand, using the weakly nonlinear technique, Pedlosky (1981) obtained a bifurcation diagram of topographically forced flows in a 2-layer model. The bifurcation diagram shows multiple equilibria and a catastrophe between them. One of the two stable equilibria corresponds to a blocked flow, and the other to a nearly zonal one.

In Pedlosky (1991), the dissipation was taken to be a sum of Ekman dampings on the upper and lower boundaries and an interfacial friction between the upper and lower layers. In this note, it is examined whether the bifurcation diagram of Pedlosky (1981) is dependent upon the form of dissipation. In particular, the following 2 cases are examined. (1) The dissipation is proportional to the potential vorticity, and (2) the dissipation is proportional to the Laplacian of the streamfunction, i.e., Ekman dampings on the upper and lower boundaries.

The organization of this note is as follows. In section 2 , the governing equation is presented. In sections 3 and 4 , the linear baroclinic and topographic problems are reviewed, respectively. In section 5 , the weakly nonlinear evolution equations are derived. In section 6 , steady solutions are calculated. In section 7 , stability properties of the steady solutions are investigated. In section 8 , concluding remarks are given.

\section{Governing equation}

The model is governed by the quasigeostrophic 2-layer potential vorticity equation in a $\beta$ channel $\{(\mathrm{x}, \mathrm{y}): 0 \leqq \mathrm{y} \leqq \mathrm{Y}, 0 \leqq \mathrm{x} \leqq \mathrm{X}$ and cyclic in $\mathrm{x}\}$,

$$
\begin{aligned}
& (\partial / \partial \mathrm{t}) \Sigma_{\mathrm{j}} \mathrm{Q}_{\mathrm{ij}}(\Delta) \psi_{\mathrm{j}}+\mathrm{J}\left(\phi_{\mathrm{i}}, \Sigma_{\mathrm{j}} \mathrm{Q}_{\mathrm{ij}}(\Delta) \psi_{\mathrm{j}}+\beta \mathrm{y}+\mathrm{h}_{\mathrm{i}}\right) \\
& +\mathrm{r} \Sigma_{\mathrm{j}} \mathrm{D}_{\mathrm{ij}}(\Delta) \psi_{\mathrm{j}}=\mathrm{f}_{\mathrm{i}},
\end{aligned}
$$

with boundary conditions for the streamfunc- tion $\psi_{\mathrm{i}}, \partial \psi_{\mathrm{i}} / \partial \mathrm{x}=0$ on $\mathrm{y}=0, \mathrm{Y}$, where $\mathrm{i}=1$ and 2 denote the upper and lower layers, respectively. $\mathrm{Q}_{\mathrm{ij}}(\Delta)$ is a symmetric $2 \times 2$ matrix given by

$$
\mathrm{Q}_{\mathrm{ij}}(\Delta)=\Delta \delta_{\mathrm{ij}}+\mathrm{F}_{\mathrm{ij}}, \delta_{\mathrm{ij}}=\left[\begin{array}{ll}
1 & 0 \\
0 & 1
\end{array}\right], \mathrm{F}_{\mathrm{ij}}=\mathrm{F}\left[\begin{array}{cc}
-1 & 1 \\
1 & -1
\end{array}\right]
$$

where $\Delta$ is the horizontal Laplacian, and $F$ is the Froude number. $r$ is the coefficient of dissipation, and the $2 \times 2$ matrix $\mathrm{D}_{\mathrm{ij}}(\Delta)$ determines the form of dissipation.

In the absence of the topography, that is, $\mathrm{h}_{\mathrm{i}}(\mathrm{x}, \mathrm{y})=0$, the external force $\mathrm{f}_{\mathrm{i}}$ induces $\mathrm{a}$ steady zonal flow $\Psi_{\mathrm{i}}(\mathrm{y})$ which satisfies

$$
\mathrm{r} \sum_{\mathrm{j}} \mathrm{D}_{\mathrm{ij}}\left(\mathrm{d}^{2} / \mathrm{dy}^{2}\right) \Psi_{\mathrm{j}}(\mathrm{y})=\mathrm{f}_{\mathrm{i}}
$$

The force $f_{i}$ is assumed to be such that the forced zonal flow $\mathrm{U}_{\mathrm{i}}=-\mathrm{d} \Psi_{\mathrm{i}}(\mathrm{y}) / \mathrm{dy}$ is independent of $y$. In the presence of the topography, we write the solution to Eq. (2-1) as

$$
\psi_{\mathrm{i}}=\Psi_{\mathrm{i}}+\psi_{\mathrm{i}}^{\prime} .
$$

Then the evolution equation of $\psi_{\mathrm{i}}{ }^{\prime}$ is derived from Eq. (2-1) as follows :

$$
\begin{aligned}
& (\partial / \partial \mathrm{t}) \Sigma_{\mathrm{j}} \mathrm{Q}_{\mathrm{ij}}(\Delta) \psi_{\mathrm{j}}{ }^{\prime}+\mathrm{J}\left(\Psi_{\mathrm{i}}, \Sigma_{\mathrm{j}} \mathrm{Q}_{\mathrm{ij}}(\Delta) \psi_{\mathrm{j}}^{\prime}+\mathrm{h}_{\mathrm{i}}\right) \\
& +\mathrm{J}\left({\psi_{\mathrm{i}}}^{\prime}, \Sigma_{\mathrm{j}} \mathrm{Q}_{\mathrm{ij}}(\Delta) \Psi_{\mathrm{j}}+\beta \mathrm{y}\right) \\
& +\mathrm{J}\left({\psi_{\mathrm{i}}}^{\prime}, \Sigma_{\mathrm{j}} \mathrm{Q}_{\mathrm{ij}}(\Delta) \psi_{\mathrm{j}}{ }^{\prime}+\mathrm{h}_{\mathrm{i}}\right)+\mathrm{r} \Sigma_{\mathrm{j}} \mathrm{D}_{\mathrm{ij}}(\Delta) \psi_{\mathrm{j}}{ }^{\prime}=0 .
\end{aligned}
$$

\section{Linear baroclinic problem}

On the assumption that the topography is small, the linearized equation of Eq. $(2-4)$ is given by

$$
\begin{aligned}
& (\partial / \partial \mathrm{t}) \Sigma_{\mathrm{j}} \mathrm{Q}_{\mathrm{ij}}(\Delta) \psi_{\mathrm{j}}{ }^{\prime}+\mathrm{r} \Sigma_{\mathrm{j}} \mathrm{D}_{\mathrm{ij}}(\Delta) \psi_{\mathrm{j}}{ }^{\prime}+\mathrm{U}_{\mathrm{i}}\left(\partial \mathrm{h}_{\mathrm{i}} / \partial \mathrm{x}\right) \\
& +\mathrm{U}_{\mathrm{i}}(\partial / \partial \mathrm{x}) \Sigma_{\mathrm{j}} \mathrm{Q}_{\mathrm{ij}}(\Delta) \psi_{\mathrm{j}}{ }^{\prime}+\beta\left(\partial \psi_{\mathrm{i}}{ }^{\prime} / \partial \mathrm{x}\right) \\
& -\Sigma_{\mathrm{j}} \mathrm{F}_{\mathrm{ij}} \mathrm{U}_{\mathrm{j}}\left(\partial \psi_{\mathrm{i}}{ }^{\prime} / \partial \mathrm{x}\right)=0 .
\end{aligned}
$$

In the absence of the topography, Eq. (3-1) becomes a partial differential equation of $\psi_{\mathrm{i}}^{\prime}$ with constant coefficients,

$$
\begin{aligned}
& (\partial / \partial \mathrm{t}) \Sigma_{\mathrm{j}} \mathrm{Q}_{\mathrm{ij}}(\Delta) \psi_{\mathrm{j}}{ }^{\prime}+\mathrm{r} \Sigma_{\mathrm{j}} \mathrm{D}_{\mathrm{ij}}(\Delta) \psi_{\mathrm{j}}{ }^{\prime} \\
& +\mathrm{U}_{\mathrm{i}}(\partial / \partial \mathrm{x}) \Sigma_{\mathrm{j}} \mathrm{Q}_{\mathrm{ij}}(\Delta) \psi_{\mathrm{j}}{ }^{\prime}+\beta\left(\partial \psi_{\mathrm{i}}{ }^{\prime} / \partial \mathrm{x}\right) \\
& -\Sigma_{\mathrm{j}} \mathrm{F}_{\mathrm{ij}} \mathrm{U}_{\mathrm{j}}\left(\partial \psi_{\mathrm{i}}{ }^{\prime} / \partial \mathrm{x}\right)=0 .
\end{aligned}
$$


In this case, we can assume that $\psi_{\mathrm{i}}{ }^{\prime}$ has the following form

$$
\begin{aligned}
& \psi_{\mathrm{i}}{ }^{\prime}=\mathrm{A}_{\mathrm{i}} \exp (\mathrm{ikx}) \operatorname{sinly}+\text { c.c., } \\
& \mathrm{k}=2 \mathrm{~m} \pi / X, \quad \mathrm{l}=\mathrm{n} \pi / \mathrm{Y}, \quad \mathrm{m}, \mathrm{n}=1,2,3, \cdots .
\end{aligned}
$$

Substituting Eq. (3-3) into Eq. (3-2), we obtain the evolution equation of $A_{i}$ as follows :

$$
\begin{aligned}
& \sum_{\mathrm{j}} \mathrm{Q}_{\mathrm{ij}}\left(-\mathrm{a}^{2}\right) \mathrm{dA} \mathrm{A}_{\mathrm{j}} / \mathrm{dt}+\mathrm{r} \Sigma_{\mathrm{j}} \mathrm{D}_{\mathrm{ij}}\left(-\mathrm{a}^{2}\right) \mathrm{A}_{\mathrm{j}} \\
& +\mathrm{ikU} \Sigma_{\mathrm{j}} \mathrm{P}_{\mathrm{ij}}\left(-\mathrm{a}^{2}\right) \mathrm{A}_{\mathrm{j}}=0,
\end{aligned}
$$

where we write $\mathrm{U}_{\mathrm{i}}=\mathrm{Uu}_{\mathrm{i}}, \mathrm{k}^{2}+\mathrm{l}^{2}=\mathrm{a}^{2}$ and

$$
\begin{aligned}
& Q_{i j}\left(-a^{2}\right)=\left[\begin{array}{ll}
-a^{2}-F & F \\
F & -a^{2}-F
\end{array}\right], \quad(3-5) \\
& P_{i j}\left(-a^{2}\right)=\left[\begin{array}{ll}
-a^{2} u_{1}-F u_{2}+\beta / U & F u_{1} \\
F u_{2} & -a^{2} u_{2}-F u_{1}+\beta / U
\end{array}\right] .
\end{aligned}
$$

With $\mathrm{r} \ll 1, \mathrm{~A}_{\mathrm{i}}$ can grow only when the matrix $\mathrm{ikU} \Sigma_{\mathrm{j}} \mathrm{Q}_{\mathrm{ij}}\left(-\mathrm{a}^{2}\right)^{-1} \mathrm{P}_{\mathrm{jk}}\left(-\mathrm{a}^{2}\right)$ has negative real eigenvalues. From Eq. (3-5) and Eq. (3-6), the eigenvalues $\sigma$ of this matrix are given by the following equation :

$$
\begin{aligned}
& \sigma=\mathrm{ikU}\left(\mathrm{u}_{1}+\mathrm{u}_{2}\right) / 2 \\
& -\mathrm{ik} \beta\left\{\left(\mathrm{a}^{2}+\mathrm{F}\right) / \mathrm{a}^{2}\left(\mathrm{a}^{2}+2 \mathrm{~F}\right)\right\} \\
& \pm \mathrm{ik}\left[\mathrm{a}^{4}\left(\mathrm{a}^{4}-4 \mathrm{~F}^{2}\right) \mathrm{U}^{2}\left(\mathrm{u}_{1}-\mathrm{u}_{2}\right)^{2}+4 \mathrm{~F}^{2} \beta^{2}\right]^{1 / 2} \\
& /\left\{2 \mathrm{a}^{2}\left(\mathrm{a}^{2}+2 \mathrm{~F}\right)\right\} .
\end{aligned}
$$

Eq. (3-7) implies that the waves grow only when

$$
\begin{aligned}
& a^{2}<2 F \\
& \text { and } U\left(u_{1}-u_{2}\right)>U_{c}=2 \beta F /\left\{a^{2}\left(4 F^{2}-a^{4}\right)^{1 / 2}\right\}
\end{aligned}
$$

The possible minimal value of $\mathrm{U}_{\mathrm{c}}$ is

$$
\mathrm{U}_{\mathrm{cmin}}=\beta / \mathrm{F} \text {. }
$$

\section{Linear topographic problem}

In this note, we consider such a topography that is given by

$$
h_{i}(x, y)=H_{i}\{\exp (i k x)+\exp (-i k x)\} \text { sinly, }
$$

where $\mathrm{k}=2 \pi \mathrm{m} / \mathrm{X}, \mathrm{l}=\pi \mathrm{n} / \mathrm{Y}, \mathrm{m}, \mathrm{n}=1,2,3, \cdots$ and $\mathrm{H}_{\mathrm{i}}$ $=\delta_{i 2} \mathrm{H}$. If the magnitude of the basic zonal flow is less than the minimal value, i.e., $\mathrm{U}\left(\mathrm{u}_{1}-\mathrm{u}_{2}\right)<\mathrm{U}_{\mathrm{cmin}}$, then there is no growing wave component except for the projection on the bases $\exp ( \pm \mathrm{ikx})$ sinly. Hence we consid er only such a disturbance that

$$
\psi_{\mathrm{i}}{ }^{\prime}=\mathrm{A}_{\mathrm{i}} \exp (\mathrm{ikx}) \operatorname{sinly}+\text { c.c.. }
$$

By sustituting Eq. (4-2) into Eq. (3-1), the evolution equation of $A_{i}$ is obtained as

$$
\begin{aligned}
& \mathrm{dA} / \mathrm{dt}+\mathrm{r} \Sigma_{\mathrm{j}} \Sigma_{\mathrm{k}} \mathrm{Q}_{\mathrm{ij}}\left(-\mathrm{a}^{2}\right)^{-1} \mathrm{D}_{\mathrm{jk}}\left(-\mathrm{a}^{2}\right) \mathrm{A}_{\mathrm{k}} \\
& +\mathrm{ikU} \Sigma_{\mathrm{j}} \Sigma_{\mathrm{k}} \mathrm{Q}_{\mathrm{ij}}\left(-\mathrm{a}^{2}\right)^{-1} \mathrm{P}_{\mathrm{jk}}\left(-\mathrm{a}^{2}\right) \mathrm{A}_{\mathrm{k}} \\
& =-\mathrm{ikUH} \Sigma_{\mathrm{j}} \mathrm{Q}_{\mathrm{ij}}\left(-\mathrm{a}^{2}\right)^{-1} \delta_{\mathrm{j} 2} \mathrm{u}_{2},
\end{aligned}
$$

where $a^{2}=k^{2}+l^{2}$. With $r>0$, the disturbance will approach a steady state. If $r$ is sufficiently small and the magnitude of the basic zonal flow $U$ is such that the matrix $i k U \Sigma_{j} Q_{i j}\left(-a^{2}\right)^{-1} P_{j k}\left(-a^{2}\right)$ has a zero eigenvalue, then a steady wave which has the same vertical structure as the corresponding right zero-eigenvector will eventually grow up. From Eq. (3-7), the matrix $\mathrm{ikU} \Sigma_{\mathrm{j}} \mathrm{Q}_{\mathrm{ij}}\left(-\mathrm{a}^{2}\right)^{-1} \mathrm{P}_{\mathrm{jk}}\left(-\mathrm{a}^{2}\right)$ has a zero eigenvalue when

$$
\begin{aligned}
& \beta / \mathrm{U}=(1 / 2)\left(\mathrm{a}^{2}+\mathrm{F}\right)\left(\mathrm{u}_{1}+\mathrm{u}_{2}\right)+\left[\mathrm{F}^{2}\left(\mathrm{u}_{1}+\mathrm{u}_{2}\right)^{2}\right. \\
& \left.+\mathrm{a}^{2}\left(\mathrm{a}^{2}-2 \mathrm{~F}\right)\left(\mathrm{u}_{1}-\mathrm{u}_{2}\right)^{2}\right]^{1 / 2} / 2, \\
& \mathrm{a}^{2} \geqq 2 \mathrm{~F},
\end{aligned}
$$

with the corresponding right eigenvector

$$
\begin{aligned}
& \mathrm{r}_{\mathrm{i}} \propto\left[\begin{array}{l}
\mathrm{Fu} \mathrm{u}_{1} \\
\mathrm{a}^{2} \mathrm{u}_{1}+\mathrm{Fu} \mathrm{u}_{2}-\beta / \mathrm{U}
\end{array}\right] \\
& \propto\left[\begin{array}{l}
\mathrm{a}^{2} \mathrm{u}_{2}+\mathrm{Fu} \mathrm{u}_{1}-\beta / \mathrm{U} \\
\mathrm{Fu}_{2}
\end{array}\right]
\end{aligned}
$$

From Eq. (3-9), the baroclinic stability condition becomes $U\left(u_{1}-u_{2}\right)<\beta / F$. It is easily seen that the linear resonant value $U$ given by Eq. (4-4) satisfies this condition, $\beta / U>$ $\mathrm{F}\left(\mathrm{u}_{1}-\mathrm{u}_{2}\right)$.

\section{Weakly nonlinear problem}

In the following, we consider a case in 
which the magnitude of the basic zonal flow is nearly equal to the resonant value of Eq. (4-4), and denote it by

$$
(1+\eta) \mathrm{U}, \quad|\eta| \ll 1,
$$

where $\mathrm{U}$ is given by Eq. (4-4). Further let it be supposed that

$$
\psi_{\mathrm{i}}^{\prime}=\mathrm{O}(\varepsilon), \quad \eta=\mathrm{O}\left(\varepsilon^{2}\right), \quad|\varepsilon| \ll 1 .
$$

If we wish to describe the system with one time scale, then from Eq. (4-3),

$$
\partial / \partial \mathrm{t}=\varepsilon^{2} \partial / \partial \tau, \mathrm{r}=\varepsilon^{2} \mathrm{r}^{(2)}, \mathrm{H}=\varepsilon^{3} \mathrm{H}^{(3)},
$$

where $\partial / \partial \tau, \mathrm{r}^{(2)}$ and $\mathrm{H}^{(3)}$ are of $\mathrm{O}(1)$, and then Eq. $(2-4)$ bacomes

$$
\begin{aligned}
& \varepsilon^{2}(\partial / \partial \tau) \Sigma_{\mathrm{j}} \mathrm{Q}_{\mathrm{ij}}(\Delta) \psi_{\mathrm{j}}{ }^{\prime}+\varepsilon^{2} \mathrm{r}^{(2)} \Sigma_{\mathrm{j}} \mathrm{D}_{\mathrm{ij}}(\Delta) \psi_{\mathrm{j}}{ }^{\prime} \\
& +\beta\left(\partial{\psi_{\mathrm{i}}}^{\prime} / \partial \mathrm{x}\right) \\
& +\left(1+\varepsilon^{2} \eta^{(2)}\right) \mathrm{U}(\partial / \partial \mathrm{x}) \Sigma_{\mathrm{j}} \mathrm{u}_{\mathrm{i}} \mathrm{Q}_{\mathrm{ij}}(\Delta) \psi_{\mathrm{j}}{ }^{\prime} \\
& -\left(1+\varepsilon^{2} \eta^{(2)}\right) \mathrm{U} \Sigma_{\mathrm{j}} \mathrm{F}_{\mathrm{ij}} \mathrm{u}_{\mathrm{j}}\left(\partial{\psi_{\mathrm{i}}}^{\prime} / \partial \mathrm{x}\right) \\
& +\varepsilon^{3}\left(1+\varepsilon^{2} \eta^{(2)}\right) \mathrm{Uu}_{\mathrm{i}}\left(\partial \mathrm{h}_{\mathrm{i}}^{(3)} / \partial \mathrm{x}\right) \\
& +\mathrm{J}\left({\psi_{\mathrm{i}}}^{\prime}, \Sigma_{\mathrm{j}} \mathrm{Q}_{\mathrm{ij}}(\Delta) \psi_{\mathrm{j}}{ }^{\prime}+\varepsilon^{3} \mathrm{~h}_{\mathrm{i}}^{(3)}\right)=0 .
\end{aligned}
$$

We expand the disturbance $\psi_{i}^{\prime}$ as

$$
\psi_{\mathrm{i}}{ }^{\prime}=\varepsilon{\phi_{\mathrm{i}}}^{(1)}+\varepsilon^{2}{\psi_{\mathrm{i}}}^{(2)}+\varepsilon^{3}{\psi_{\mathrm{i}}}^{(3)}+\cdots,
$$

substitute this expansion into Eq. (5-4), and equate the terms of the like order of $\varepsilon$. First, the $O(\varepsilon)$ equation becomes

$$
\Sigma_{\mathrm{j}} \mathrm{L}_{\mathrm{ij}}(\partial / \partial \mathrm{x}, \partial / \partial \mathrm{y}) \psi_{\mathrm{j}}^{(1)}=0
$$

where $\mathrm{L}_{\mathrm{ij}}(\partial / \partial \mathrm{x}, \partial / \partial \mathrm{y})$

$$
=\mathrm{U}(\partial / \partial \mathrm{x})\left[\mathrm{u}_{\mathrm{i}} \mathrm{Q}_{\mathrm{ij}}(\Delta)+\delta_{\mathrm{ij}}\left\{(\beta / \mathrm{U})-\Sigma_{\mathrm{k}} \mathrm{F}_{\mathrm{ik}} \mathrm{u}_{\mathrm{k}}\right\}\right] \text {. }
$$

Since the magnitude of the basic zonal flow is nearly equal to the linear resonant value, we take as the solution of Eq. (5-6)

$$
\psi_{\mathrm{i}}^{(1)}=\mathrm{r}_{\mathrm{i}} \mathrm{A}(\tau) \exp (\mathrm{ikx}) \text { sinly }+ \text { c.c., }
$$

where the amplitude $r_{i} A(\tau)$ is proportional to the right eigenvector $r_{i}$ in Eq. (4-5), and thereby the linear operator in Eq. (5-7) becomes

$$
\begin{aligned}
& \mathrm{L}_{\mathrm{ij}}(\mathrm{ik}, \mathrm{il})=\mathrm{ikU}\left[\mathrm{u}_{\mathrm{i}} \mathrm{Q}_{\mathrm{ij}}\left(-\mathrm{a}^{2}\right)\right. \\
& \left.+\delta_{\mathrm{ij}}\left\{(\beta / \mathrm{U})-\Sigma_{\mathrm{k}} \mathrm{F}_{\mathrm{ik}} \mathrm{U}_{\mathrm{k}}\right\}\right]=\mathrm{ikUP} \mathrm{P}_{\mathrm{ij}}\left(-\mathrm{a}^{2}\right) .
\end{aligned}
$$

Next, the $O\left(\varepsilon^{2}\right)$ equation becomes

$$
\Sigma_{\mathrm{j}} \mathrm{L}_{\mathrm{ij}}(\partial / \partial \mathrm{x}, \partial / \partial \mathrm{y}) \psi_{\mathrm{j}}^{(2)}=0 .
$$

Redefining $\psi_{\mathrm{i}}{ }^{(1)}$, we can take the following solution to Eq. (5-9)

$$
\psi_{\mathrm{i}}^{(2)}=\psi_{\mathrm{i}}^{(2)}(\mathrm{y}, \tau) \text {. }
$$

Next, the $O\left(\varepsilon^{3}\right)$ equation becomes

$$
\begin{aligned}
& \sum_{\mathrm{j}} \mathrm{L}_{\mathrm{ij}}(\partial / \partial \mathrm{x}, \partial / \partial \mathrm{y}) \psi_{\mathrm{j}}^{(3)}+\mathrm{Uu}_{\mathrm{i}}\left(\partial \mathrm{h}_{\mathrm{i}}^{(3)} / \partial \mathrm{x}\right) \\
& +(\partial / \partial \tau) \Sigma_{\mathrm{j}} \mathrm{Q}_{\mathrm{ij}}(\Delta) \psi_{\mathrm{j}}^{(1)}+\mathrm{r}^{(2)} \Sigma_{\mathrm{j}} \mathrm{D}_{\mathrm{ij}}(\Delta) \psi_{\mathrm{j}}^{(1)} \\
& +\Sigma_{\mathrm{j}} \eta^{(2)} \mathrm{U}\left\{\partial \mathrm{L}_{\mathrm{ij}}(\partial / \partial \mathrm{x}, \partial / \partial \mathrm{y}) / \partial \mathrm{U}\right\} \psi_{\mathrm{j}}^{(1)} \\
& +\mathrm{J}\left(\psi_{\mathrm{i}}^{(1)}, \Sigma_{\mathrm{j}} \mathrm{Q}_{\mathrm{ij}}(\Delta) \psi_{\mathrm{j}}^{(2)}\right)+\mathrm{J}\left(\phi_{\mathrm{i}}^{(2)}, \Sigma_{\mathrm{j}} \mathrm{Q}_{\mathrm{ij}}(\Delta) \psi_{\mathrm{j}}^{(1)}\right) \\
& =0 .
\end{aligned}
$$

Here we introduce the left zero-eigenvector of $P_{i j}\left(-a^{2}\right)$ which can be written as $l_{i}=r_{i} / u_{i}$ and normalized as $\Sigma_{\mathrm{i}} \mathrm{l}_{\mathrm{i}} \mathrm{r}_{\mathrm{i}}=1$. According to the procedure to remove the secular terms, multiplying Eq. (5-11) by $l_{i} \exp (-\mathrm{ikx})$ sinly, and taking the summation with respect to $\mathrm{i}$ and integration with respect to $(x, y)$ in $\{0 \leqq x \leqq X, 0$ $\leqq \mathrm{y} \leqq \mathrm{Y}\}$, we obtain the following evolution equation for $\mathrm{A}$ :

$$
\begin{aligned}
& \text { QdA } / \mathrm{d} \tau+\mathrm{r}^{(2)} \mathrm{DA}-\mathrm{ik} \beta \eta^{(2)} \mathrm{A}+\mathrm{ikUH} \mathrm{H}^{(3)} \\
& +\mathrm{iklA} \Sigma_{\mathrm{i}} \mathrm{E}_{\mathrm{i}} \Phi_{\mathrm{i}}=0 \\
& \text { where } \mathrm{Q}=\Sigma_{\mathrm{i}} \Sigma_{\mathrm{j}} \mathrm{l}_{\mathrm{i}} \mathrm{Q}_{\mathrm{ij}}\left(-\mathrm{a}^{2}\right) \mathrm{r}_{\mathrm{j}}, \\
& \qquad \begin{aligned}
\mathrm{D} & =\Sigma_{\mathrm{i}} \Sigma_{\mathrm{j}} \mathrm{l}_{\mathrm{i}} \mathrm{D}_{\mathrm{ij}}\left(-\mathrm{a}^{2}\right) \mathrm{r}_{\mathrm{j}} \\
\text { and } \quad \Phi_{\mathrm{i}} & =(2 / \mathrm{Y}) \int_{0} \mathrm{Y} \mathrm{dy} \sin 2 \mathrm{ly} \psi_{\mathrm{i}}^{(2)}, \\
\mathrm{E}_{\mathrm{i}} & =\Sigma_{\mathrm{j}}\left\{\mathrm{r}_{\mathrm{j}} \mathrm{Q}_{\mathrm{ji}}\left(-\mathrm{a}^{2}\right) \mathrm{l}_{\mathrm{i}}-\mathrm{l}_{\mathrm{j}} \mathrm{r}_{\mathrm{j}} \mathrm{Q}_{\mathrm{li}}\left(-4 \mathrm{l}^{2}\right)\right\}
\end{aligned}
\end{aligned}
$$

Next, the $O\left(\varepsilon^{4}\right)$ equation becomes

$$
\begin{aligned}
& \Sigma_{\mathrm{j}} \mathrm{L}_{\mathrm{ij}}(\partial / \partial \mathrm{x}, \partial / \partial \mathrm{y}) \psi_{\mathrm{j}}^{(4)} \\
& +\Sigma_{\mathrm{j}} \eta^{(2)} \mathrm{U}\left\{\partial \mathrm{L}_{\mathrm{ij}}(\partial / \partial \mathrm{x}, \partial / \partial \mathrm{y}) / \partial \mathrm{U}\right\} \psi_{\mathrm{j}}^{(2)} \\
& +(\partial / \partial \tau) \Sigma_{\mathrm{j}} \mathrm{Q}_{\mathrm{ij}}(\Delta) \psi_{\mathrm{j}}^{(2)}+\mathrm{r}^{(2)} \Sigma_{\mathrm{j}} \mathrm{D}_{\mathrm{ij}}(\Delta) \psi_{\mathrm{j}}^{(2)} \\
& +\mathrm{J}\left(\phi_{\mathrm{i}}^{(1)}, \mathrm{h}_{\mathrm{i}}^{(3)}\right)+\mathrm{J}\left(\psi_{\mathrm{i}}^{(1)}, \Sigma_{\mathrm{j}} \mathrm{Q}_{\mathrm{ij}}(\Delta) \psi_{\mathrm{j}}^{(3)}\right) \\
& +\mathrm{J}\left(\phi_{\mathrm{i}}^{(3)}, \Sigma_{\mathrm{j}} \mathrm{Q}_{\mathrm{ij}}(\Delta) \psi_{\mathrm{j}}^{(1)}\right)=0 .
\end{aligned}
$$

Integrating Eq. (5-15) with respect to $\mathrm{x}$ yields

$$
\begin{aligned}
& (\partial / \partial \tau) \Sigma_{\mathrm{j}} \mathrm{Q}_{\mathrm{ij}}(\Delta) \psi_{\mathrm{j}}^{(2)}+\mathrm{r}^{(2)} \Sigma_{\mathrm{j}} \mathrm{D}_{\mathrm{ij}}(\Delta) \psi_{\mathrm{j}}^{(2)} \\
& +\mathrm{ik} \mathrm{r}_{\mathrm{i}} \mathrm{H}_{\mathrm{i}}^{(3)}(\mathrm{A}-\overline{\mathrm{A}}) \sin 2 \mathrm{ly} \\
& -(1 / \mathrm{X}) \int_{0}^{\mathrm{x}} \mathrm{dx}(\partial / \partial \mathrm{y})\left[\left(\psi_{\mathrm{i}}^{(1)} / \mathrm{Uu}_{\mathrm{i}}\right) \Sigma_{\mathrm{j}} \mathrm{L}_{\mathrm{ij}}(\partial / \partial \mathrm{x}, \partial / \partial \mathrm{y}) \psi_{\mathrm{j}}^{(3)}\right]
\end{aligned}
$$




$$
=0 \text {. }
$$

On the other hand, multiplying Eq. (5-11) by $\psi_{i}^{(1)} / \mathrm{Uu}_{\mathrm{i}}$, integrating with respect to $\mathrm{x}$ and differentiating with respect to y yields

$$
\begin{aligned}
& (1 / \mathrm{X}) \int_{0} \mathrm{x} d \mathrm{x}(\partial / \partial \mathrm{y})\left[\left(\psi_{\mathrm{i}}^{(1)} / \mathrm{Uu} \mathrm{u}_{\mathrm{i}}\right) \Sigma_{\mathrm{j}} \mathrm{L}_{\mathrm{ij}}(\partial / \partial \mathrm{x}, \partial / \partial \mathrm{y}) \psi_{\mathrm{j}}^{(3)}\right. \\
& +\mathrm{ikl}(\overline{\mathrm{A}}-\mathrm{A}) \mathrm{r}_{\mathrm{i}} \mathrm{H}_{\mathrm{i}}^{(3)} \sin 2 \mathrm{ly} \\
& +(1 / \mathrm{U})\left(\mathrm{r}_{\mathrm{i}} / \mathrm{u}_{\mathrm{i}}\right) \Sigma_{\mathrm{j}} \mathrm{Q}_{\mathrm{ij}}\left(-\mathrm{a}^{2}\right) \mathrm{r}_{\mathrm{j}}\{\mathrm{A}(\mathrm{d} \overline{\mathrm{A}} / \mathrm{d} \tau) \\
& +(\mathrm{dA} / \mathrm{d} \tau) \overline{\mathrm{A}} / \sin 21 \mathrm{y} \\
& +\left(2 \mathrm{lr} \mathrm{r}^{(2)} / \mathrm{U}\right)\left(\mathrm{r}_{\mathrm{i}} / \mathrm{u}_{\mathrm{i}}\right) \Sigma_{\mathrm{j}} \mathrm{D}_{\mathrm{ij}}\left(-\mathrm{a}^{2}\right) \mathrm{r}_{\mathrm{j}} \mathrm{A} \overline{\mathrm{A}} \sin 2 \mathrm{ly}=0
\end{aligned}
$$

Adding Eq. (5-16) to Eq. (5-17), multiplying by sin2ly, integrating with respect to $\mathrm{y}$, and further eliminating $\mathrm{A}(\mathrm{dA} / \mathrm{d} \tau)+(\mathrm{d} \overline{\mathrm{A}} / \mathrm{d} \tau) \overline{\mathrm{A}}$ by using Eq. (5-12), we obtain the following evolution equation for $\Phi_{1}$

$$
\begin{aligned}
& \sum_{\mathrm{j}} \mathrm{Q}_{\mathrm{ij}}\left(-41^{2}\right)\left(\mathrm{d} \Phi_{\mathrm{j}} / \mathrm{d} \tau\right)+\mathrm{r}^{(2)} \Sigma_{\mathrm{j}} \mathrm{D}_{\mathrm{ij}}\left(-4 \mathrm{l}^{2}\right) \Phi_{\mathrm{j}} \\
& +\mathrm{i}\left(\mathrm{klH} \mathrm{H}^{(3)} / \mathrm{Q}\right)\left(\mathrm{r}_{\mathrm{i}} / \mathrm{u}_{\mathrm{i}}\right) \Sigma_{\mathrm{j}} \mathrm{Q}_{\mathrm{ij}}\left(-\mathrm{a}^{2}\right) \mathrm{r}_{\mathrm{j}}(\mathrm{A}-\overline{\mathrm{A}}) \\
& +\left(21 \mathrm{r}^{(2)} / \mathrm{U}\right)\left(\mathrm{r}_{\mathrm{i}} / \mathrm{u}_{\mathrm{i}}\right) \Sigma_{\mathrm{j}}\left\{\mathrm{D}_{\mathrm{ij}}\left(-\mathrm{a}^{2}\right)-(\mathrm{D} / \mathrm{Q}) \mathrm{Q}_{\mathrm{ij}}\left(-\mathrm{a}^{2}\right)\right\} \mathrm{r}_{\mathrm{j}} \mathrm{AA} \\
& =0 .
\end{aligned}
$$

If we write the real and imaginary parts of $A$ explicitly, then from Eq. (5-12) and Eq. (5-18) the evolution equations become

$$
\begin{aligned}
& \mathrm{dReA} / \mathrm{d} \tau+\mathrm{rReA}-\eta \operatorname{ImA}+\operatorname{ImA} \Sigma_{\mathrm{i}} \mathrm{E}_{\mathrm{i}} \mathrm{B}_{\mathrm{i}}=0, \\
& \mathrm{dImA} / \mathrm{d} \tau+\mathrm{rImA}+\eta \operatorname{ReA}-\operatorname{ReA} \Sigma_{\mathrm{i}} \mathrm{E}_{\mathrm{i}} \mathrm{B}_{\mathrm{i}}=\mathrm{h}, \\
& \mathrm{dB}_{\mathrm{i}} / \mathrm{d} \tau+\mathrm{r} \Sigma_{\mathrm{j}} \mathrm{M}_{\mathrm{ij}} \mathrm{B}_{\mathrm{j}}+\mathrm{hN}_{\mathrm{i}} \operatorname{ImA}+\mathrm{r} \omega_{\mathrm{i}}\left\{\mathrm{ReA}^{2}+\right. \\
& \left.\mathrm{ImA}^{2}\right\}=0 \\
& \text { where } \quad \mathrm{r}=\mathrm{r}^{(2)}(\mathrm{D} / \mathrm{Q}), \quad \eta=-\eta^{(2)}(\mathrm{k} \beta / \mathrm{Q}), \\
& \mathrm{h}=-\mathrm{H}^{(3)} \mathrm{kU} / \mathrm{Q}, \quad \mathrm{B}_{\mathrm{i}}=-\Phi_{\mathrm{i}}(\mathrm{kl} / \mathrm{Q}) \\
& \text { and } \quad \mathrm{M}_{\mathrm{ij}}=(\mathrm{Q} / \mathrm{D}) \Sigma_{\mathrm{k}} \mathrm{Q}_{\mathrm{ik}}\left(-4 \mathrm{l}^{2}\right)^{-1} \mathrm{D}_{\mathrm{kj}}\left(-41^{2}\right), \\
& \mathrm{N}_{\mathrm{i}}=-2\left(\mathrm{kl} \mathrm{l}^{2} / \mathrm{UQ}\right) \Sigma_{\mathrm{j}} \Sigma_{\mathrm{k}} \mathrm{Q}_{\mathrm{ij}}\left(-4 \mathrm{l}^{2}\right)^{-1} \mathrm{l}_{\mathrm{j}} \mathrm{Q}_{\mathrm{jk}}\left(-\mathrm{a}^{2}\right) \mathrm{r}_{\mathrm{k}}, \\
& \omega_{\mathrm{i}}=-\left(2 \mathrm{kl} \mathrm{l}^{2} / \mathrm{DU}\right) \Sigma_{\mathrm{j}} \Sigma_{\mathrm{k}} \mathrm{Q}_{\mathrm{ij}}\left(-4 \mathrm{l}^{2}\right)^{-1} \mathrm{l}_{\mathrm{j}}\left\{\mathrm{D}_{\mathrm{jk}}\left(-\mathrm{a}^{2}\right)\right. \\
& \left.\quad-(\mathrm{D} / \mathrm{Q}) \mathrm{Q}_{\mathrm{jk}}\left(-\mathrm{a}^{2}\right)\right\} \mathrm{r}_{\mathrm{k}} .
\end{aligned}
$$

$A$ and $B_{i}$ represent the amplitude of the disturbance and the correction of the basic zonal flow, respectively.

\section{Steady solution}

From Eq. (5-19), any steady solution must satisfy the following equations

$$
\begin{aligned}
& r \operatorname{Re} \overline{\mathrm{A}}-\eta \operatorname{Im} \overline{\mathrm{A}}+\operatorname{Im} \overline{\mathrm{A}} \Sigma_{\mathrm{i}} \mathrm{E}_{\mathrm{i}} \overline{\mathrm{B}}_{\mathrm{i}}=0, \\
& \mathrm{r} \operatorname{Im} \overline{\mathrm{A}}+\eta \operatorname{Re} \overline{\mathrm{A}}-\operatorname{Re} \overline{\mathrm{A}} \Sigma_{\mathrm{i}} \mathrm{E}_{\mathrm{i}} \overline{\mathrm{B}}_{\mathrm{i}}=\mathrm{h}, \\
& \mathrm{r} \sum_{\mathrm{j}} \mathrm{M}_{\mathrm{ij}} \overline{\mathrm{B}}_{\mathrm{j}}+\mathrm{hN} \mathrm{N}_{\mathrm{i}} \operatorname{Im} \overline{\mathrm{A}}+\mathrm{r} \omega_{\mathrm{i}}\left\{\operatorname{Re} \overline{\mathrm{A}}^{2}+\operatorname{Im} \overline{\mathrm{A}}^{2}\right\}=0 .
\end{aligned}
$$

Writing $\sum_{\mathrm{i}} \mathrm{E}_{\mathrm{i}} \overline{\mathrm{B}}_{\mathrm{i}}=\mathrm{B}$, from Eq. (6-1) the steady solutions are given by the following equation:

$$
\begin{aligned}
& \mathrm{G}(\eta, \mathrm{B})=\mathrm{B}(\eta-\mathrm{B})^{2}+\mathrm{r}^{2} \mathrm{~B}+\mathrm{bh}^{2}=0, \\
& \operatorname{Re} \overline{\mathrm{A}}=-(1 / \mathrm{bh}) \mathrm{B}(\eta-\mathrm{B}), \operatorname{Im} \overline{\mathrm{A}}=-(\mathrm{r} / \mathrm{bh}) \mathrm{B}, \\
& \overline{\mathrm{B}}_{\mathrm{i}}=-2\left(\mathrm{kl}^{2} / \mathrm{bQU}\right) \mathrm{BS}_{\mathrm{i}}
\end{aligned}
$$

where $\mathrm{b}=-\left(2 \mathrm{kl}^{2} / \mathrm{QU}\right) \sum_{\mathrm{i}} \mathrm{E}_{\mathrm{i}} \mathrm{S}_{\mathrm{i}}$,

$$
\mathrm{S}_{\mathrm{i}}=\Sigma_{\mathrm{j}} \Sigma_{\mathrm{k}} \mathrm{D}_{\mathrm{ij}}\left(-41^{2}\right)^{-1} 1_{\mathrm{j}} \mathrm{D}_{\mathrm{jk}}\left(-\mathrm{a}^{2}\right) \mathrm{r}_{\mathrm{k}} \text {. }
$$

From Eq. (6-2), we can see that any bifurcation point lies on the following hyperbola in $(\eta, \mathrm{B})$ - plane,

$$
\partial \mathrm{G}(\eta, \mathrm{B}) / \partial \mathrm{B}=(\eta-\mathrm{B})(\eta-3 \mathrm{~B})+\mathrm{r}^{2}=0 .
$$

From Eq. (6-2) and Eq. (6-5), the steady solution bifurcation diagram is shown to be the same as Pedlosky (1981)'s except for the stability irrespective of the dissipation form $\mathrm{D}_{\mathrm{ij}}(\Delta)$ (see Fig.1).

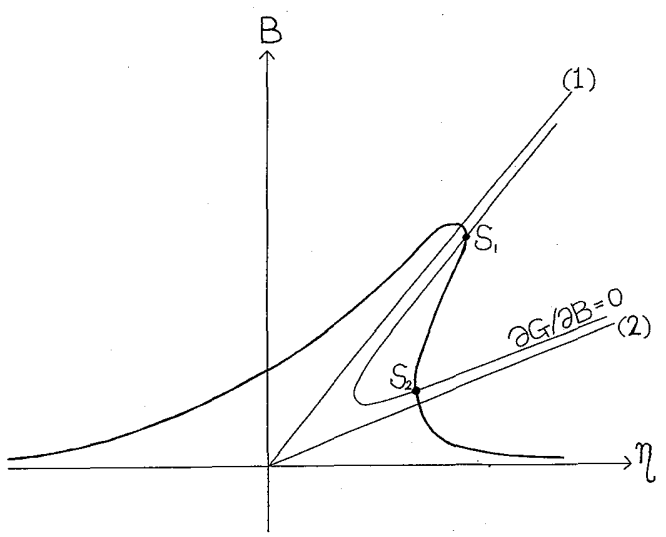

Fig. 1 Steady solution diagram (a case of $b<$ $0)$. (1) $\eta=\mathrm{B}$ and (2) $\eta=3 \mathrm{~B}$. $\mathrm{S}_{1}$ and $\mathrm{S}_{2}$ indicate steady solution bifurcation points. 


\section{Stability properties of the steady solutions}

\section{7-1. Eigenvalues determining the stability properties}

As shown in section 6 , any steady solution $\left\{\operatorname{Re} \overline{\mathrm{A}}, \operatorname{Im} \overline{\mathrm{A}}, \overline{\mathrm{B}}_{\mathrm{i}}\right\}$ belongs to a 3-dimensional sub-space spanned by $\operatorname{ReA}, \operatorname{ImA}$ and $S_{\mathrm{i}}$. The vector $S_{i}$ is independent of the control parameter $\eta$. Hereafter we denote this sub-space as $\left[\mathrm{A}, \mathrm{S}_{\mathrm{i}}\right]$. We consider a projection of Eq. $(5-19)$ on $\left[A, S_{i}\right]$. If a steady solution is unstable in $\left[A, S_{i}\right]$, then this steady solution is also unstable in the full space. Replacing $B_{i}$ by its projection $B_{i}{ }^{\prime}$ on $\left[A, S_{i}\right]$, and writing $B_{i}{ }^{\prime}$ $=\left(B / \Sigma_{j} E_{j} S_{j}\right) S_{i}$, the projection of Eq. $(5-19)$ becomes

$$
\begin{aligned}
& \mathrm{dReA} / \mathrm{d} \tau+\mathrm{rRe} A-\eta \operatorname{Im} \mathrm{A}+\mathrm{BIm} \mathrm{A}=0, \\
& \mathrm{~d} \operatorname{Im} \mathrm{A} / \mathrm{d} \tau+\mathrm{rIm} A+\eta \operatorname{ReA}-\mathrm{BReA}=\mathrm{h}, \\
& \mathrm{dB} / \mathrm{d} \tau+\mathrm{rMB}+\mathrm{hNIm} A+\mathrm{r} \omega\left\{\operatorname{ReA}^{2}+\operatorname{ImA}^{2}\right\} \\
& =0,
\end{aligned}
$$

where $\mathrm{M}=\sum_{\mathrm{i}} \Sigma_{\mathrm{j}} \mathrm{S}_{\mathrm{i}} \mathrm{M}_{\mathrm{ij}} \mathrm{S}_{\mathrm{j}} / \Sigma_{\mathrm{k}} \mathrm{S}_{\mathrm{k}} \mathrm{S}_{\mathrm{k}}$,

$$
\mathrm{N}=\Sigma_{\mathrm{i}} \mathrm{S}_{\mathrm{i}} \mathrm{N}_{\mathrm{i}} \sum_{\mathrm{j}} \mathrm{E}_{\mathrm{j}} \mathrm{S}_{\mathrm{j}} / \Sigma_{\mathrm{k}} \mathrm{S}_{\mathrm{k}} \mathrm{S}_{\mathrm{k}}
$$

and $\quad \omega=\Sigma_{\mathrm{i}} \mathrm{S}_{\mathrm{i}} \omega_{\mathrm{i}} \Sigma_{\mathrm{j}} \mathrm{E}_{\mathrm{j}} \mathrm{S}_{\mathrm{j}} / \Sigma_{\mathrm{k}} \mathrm{S}_{\mathrm{k}} \mathrm{S}_{\mathrm{k}}$

From Eq. (5-21), Eq. (6-4) and Eq. (7-2), we see that

$$
\begin{aligned}
& (\mathrm{N}+\omega) / \mathrm{b}=\mathrm{M} \\
& =(\mathrm{Q} / \mathrm{D}) \Sigma_{\mathrm{i}} \Sigma_{\mathrm{j}} \Sigma_{\mathrm{k}} \mathrm{S}_{\mathrm{i}} \mathrm{Q}_{\mathrm{ij}}\left(-4 \mathrm{l}^{2}\right)^{-1} \mathrm{l}_{\mathrm{j}} \mathrm{D}_{\mathrm{jk}}\left(-\mathrm{a}^{2}\right) \mathrm{r}_{\mathrm{k}} \\
& / \Sigma_{\mathrm{i}} \mathrm{S}_{\mathrm{i}} \mathrm{S}_{\mathrm{i}}, \\
& \mathrm{N} / \mathrm{b}=\Sigma_{\mathrm{i}} \Sigma_{\mathrm{j}} \Sigma_{\mathrm{k}} \mathrm{S}_{\mathrm{i}} \mathrm{Q}_{\mathrm{ij}}\left(-41^{2}\right)^{-1} \mathrm{l}_{\mathrm{j}} \mathrm{Q}_{\mathrm{jk}}\left(-\mathrm{a}^{2}\right) \mathrm{r}_{\mathrm{k}} \\
& / \Sigma_{\mathrm{i}} \mathrm{S}_{\mathrm{i}} \mathrm{S}_{\mathrm{i}} .
\end{aligned}
$$

Using Eq. (7-3), steady solutions of Eq. (7-1) are given by Eq. (6-2) and Eq. (6-3) as expected.

stability properties of steady solutions is determined by eigenvalues of the following matrix :

$$
\left[\begin{array}{lll}
\mathrm{r}+\sigma & -\eta+\mathrm{B} & \operatorname{ImA} \\
\eta-\mathrm{B} & \mathrm{r}+\sigma & -\operatorname{ReA} \\
2 \mathrm{r} \omega \operatorname{ReA} & \mathrm{hN}+2 \mathrm{r} \omega \operatorname{ImA} & \mathrm{rM}+\sigma
\end{array}\right]
$$

The eigenvalues $\sigma$ are given by

$$
\begin{aligned}
& \mathrm{E}(\sigma)=\sigma^{3}+\mathrm{r}(2+\mathrm{M}) \sigma^{2}+\left[(2 \mathrm{M}+1) \mathrm{r}^{2}\right. \\
& \left.+(\eta-\mathrm{B})^{2}-(\mathrm{N} / \mathrm{b}) \mathrm{B}(\eta-\mathrm{B})\right] \sigma \\
& +\mathrm{rM} \partial \mathrm{G}(\eta, \mathrm{B}) / \partial \mathrm{B}=0 .
\end{aligned}
$$

As expected, from Eq. (7-5), at least one eigenvalue denoted by $\sigma_{1}$ vanishes if and only if on the bifurcation hyperbola $\partial \mathrm{G}(\eta, \mathrm{B}) / \partial \mathrm{B}=0$,

$$
\sigma_{1}=0 \longleftrightarrow \partial \mathrm{G}(\eta, \mathrm{B}) / \partial \mathrm{B}=0 .
$$

\section{7-2. Region for $\sigma$ to be complex in $(\eta, B)$-plane}

In order to find the region for $\sigma$ to be complex, we differentiate Eq. (7-5) with respect to $\sigma$,

$$
\begin{aligned}
& \partial \mathrm{E}(\sigma) / \partial \sigma=3 \sigma^{2}+2 \mathrm{r}(2+\mathrm{M}) \sigma+(2 \mathrm{M}+1) \mathrm{r}^{2} \\
& +(\eta-\mathrm{B})^{2}-(\mathrm{N} / \mathrm{b}) \mathrm{B}(\eta-\mathrm{B}) .
\end{aligned}
$$

From Eq. $(7-7), \sigma=\sigma_{ \pm}$at which $\mathrm{E}(\sigma)$ has extrema are given by

$$
\begin{aligned}
& \sigma_{ \pm}=(1 / 3)\left[-\mathrm{r}(2+\mathrm{M}) \pm\left\{\mathrm{r}^{2}(\mathrm{M}-1)^{2}\right.\right. \\
& \left.\left.-3(\eta-\mathrm{B})^{2}+3(\mathrm{~N} / \mathrm{b}) \mathrm{B}(\eta-\mathrm{B})\right\}^{1 / 2}\right] .
\end{aligned}
$$

Substituting Eq. (7-8) into Eq. (7-5), the extrema E $\left(\sigma_{ \pm}\right)$are calculated as

$$
\begin{aligned}
& \mathrm{E}\left(\sigma_{ \pm}\right)= \pm(2 / 9)\left\{-\left(\mathrm{r}^{2} / 3\right)(\mathrm{M}-1)^{2}+(\eta-\mathrm{B})^{2}\right. \\
& -(\mathrm{N} / \mathrm{b}) \mathrm{B}(\eta-\mathrm{B})\} \cdot\left\{\mathrm{r}^{2}(\mathrm{M}-1)^{2}-3(\eta-\mathrm{B})^{2}\right. \\
& +3(\mathrm{~N} / \mathrm{b}) \mathrm{B}(\eta-\mathrm{B})\}^{1 / 2} \\
& +\left(2 \mathrm{r}^{3} / 27\right)(\mathrm{M}-1)^{3}+(2 \mathrm{r} / 3)(\mathrm{M}-1)(\eta-\mathrm{B})^{2} \\
& +\mathrm{r}\{(\mathrm{N} / 3 \mathrm{~b})(2+\mathrm{M})-2 \mathrm{M}\} \mathrm{B}(\eta-\mathrm{B}) .
\end{aligned}
$$

The function $\mathrm{D}(\eta, \mathrm{B})$ defined as

$$
\mathrm{D}(\eta, \mathrm{B})=\mathrm{E}\left(\sigma_{+}\right) \mathrm{E}\left(\sigma_{-}\right)
$$

determines the region for $\sigma$ to be complex. From Eq. $(7-9)$, the curve $\mathrm{D}(\eta, \mathrm{B})=0$ is a union of a line $\eta=\mathrm{B}$ and a curve with asymptotes $\eta=\mathrm{B}$ and $\eta=\{1+(\mathrm{N} / \mathrm{b})\} \mathrm{B}$. In the region $\mathrm{D}(\eta, \mathrm{B})>0$, there exist two complex eigenvalues denoted by $\sigma_{2,3}$,

$$
\sigma_{2,3} \text { are complex for } \mathrm{D}(\eta, \mathrm{B})>0 \text {. }
$$

For sufficiently small $r$, 
the region $\mathrm{D}(\eta, \mathrm{B})>0$

$\simeq$ the region $(\eta-\mathrm{B})[\eta-\{1+(\mathrm{N} / \mathrm{b})\} \mathrm{B}]>0$.

\section{7-3. Curve for $\operatorname{Re}\left\{\partial_{2,3}\right\}$ to vanish in $(\eta, B)$-plane}

If we explicity write $\sigma=\sigma_{\mathrm{r}}+\mathrm{i} \sigma_{\mathrm{i}}$ for complex eigenvalues, then Eq. $(7-5)$ becomes

$$
\begin{aligned}
& \sigma_{\mathrm{r}}\left[{\sigma_{\mathrm{r}}}^{2}+\mathrm{r}(2+\mathrm{M}) \sigma_{\mathrm{r}}+(2 \mathrm{M}+1) \mathrm{r}^{2}+(\eta-\mathrm{B})^{2}\right. \\
& -(\mathrm{N} / \mathrm{b}) \mathrm{B}(\eta-\mathrm{B})]+\mathrm{rM} \partial \mathrm{G}(\eta, \mathrm{B}) / \partial \mathrm{B} \\
& =\left\{3 \sigma_{\mathrm{r}}+\mathrm{r}(2+\mathrm{M})\right\} \sigma_{\mathrm{i}}{ }^{2}, \\
& 3{\sigma_{\mathrm{r}}}^{2}+2 \mathrm{r}(2+\mathrm{M}) \sigma_{\mathrm{r}}+(2 \mathrm{M}+1) \mathrm{r}^{2}+(\eta-\mathrm{B})^{2} \\
& -(\mathrm{N} / \mathrm{b}) \mathrm{B}(\eta-\mathrm{B})=\sigma_{\mathrm{i}}{ }^{2} .
\end{aligned}
$$

Substituting Eq. (7-13) into Eq. (7-12), we obtain the equation for $\sigma_{\mathrm{r}}$,

$$
\begin{aligned}
& \sigma_{\mathrm{r}}\left[4 \sigma_{\mathrm{r}}^{2}+4 \mathrm{r}(2+\mathrm{M}) \sigma_{\mathrm{r}}+(2 \mathrm{M}+1) \mathrm{r}^{2}\right. \\
& \left.+\mathrm{r}^{2}(2+\mathrm{M})^{2}+(\eta-\mathrm{B})^{2}-(\mathrm{N} / \mathrm{b}) \mathrm{B}(\eta-\mathrm{B})\right] \\
& +\mathrm{rP}(\eta, \mathrm{B})=0,
\end{aligned}
$$

where $\mathrm{P}(\eta, \mathrm{B})=(\mathrm{M}+1)^{2} \mathrm{r}^{2}$

$$
+(\eta-\mathrm{B})[\eta-\{1-\mathrm{M}+(2+\mathrm{M})(\mathrm{N} / 2 \mathrm{~b})\} \mathrm{B}] \text {. }
$$

Let it be supposed that $\sigma_{\mathrm{r}}$ is negative (positive) on and inside (outside) of the hyperbola $\mathrm{P}(\eta, \mathrm{B})=0$,

$$
\begin{aligned}
& \sigma_{\mathrm{r}}<0 \text { for } \mathrm{P}(\eta, \mathrm{B}) \leqq 0 \\
& \text { or } \sigma>0 \text { for } \mathrm{P}(\eta, \mathrm{B}) \geqq 0 .
\end{aligned}
$$

Then Eq. (7-16) implies that

$$
\begin{aligned}
& 4{\sigma_{\mathrm{r}}}^{2}+4 \mathrm{r}(2+\mathrm{M}) \sigma_{\mathrm{r}}+(2 \mathrm{M}+1) \mathrm{r}^{2}+\mathrm{r}^{2}(2+\mathrm{M})^{2} \\
& +(\eta-\mathrm{B})^{2}-(\mathrm{N} / \mathrm{b}) \mathrm{B}(\eta-\mathrm{B}) \leqq 0 .
\end{aligned}
$$

Substituting Eq.(7-17) into Eq. (7-13), we obtain

$$
\left\{\sigma_{\mathrm{r}}+\mathrm{r}(2+\mathrm{M})\right\}^{2}+\sigma_{\mathrm{i}}^{2} \leqq 0 .
$$

Eq. (7-18) contradicts the fact that $\sigma$ is complex. Therefore we can conclude that in the complex region

$$
\begin{array}{lll}
\sigma_{\mathrm{r}}>0 & \text { for } & \mathrm{P}(\eta, \mathrm{B})<0, \\
\sigma_{\mathrm{r}}<0 & \text { for } & \mathrm{P}(\eta, \mathrm{B})>0 .
\end{array}
$$

\section{Dependence on the dissipation form $D_{\mathrm{ij}}(\Delta)$}

\section{8-1. Case of dissipation proportional to potential vorticity}

If the dissipation is proportional to the potential vorticity, i.e., $\mathrm{D}_{\mathrm{ij}}(\Delta)=\mathrm{Q}_{\mathrm{ij}}(\Delta)$, then the wave-wave interaction term vanishes, that is, $\omega_{\mathrm{i}}=0$ and Eq. (5-19) can be reduced to a 3 -dimensional system. In this case, the 3-dimensional projected equations obtained above are indentical to those of the full 4-dimensional system with $\omega=0$ and $M=N / b$ $=1$. Then for sufficiently small $r$, Eqs. $(7-11)$ and (7-19) become

$\sigma_{2,3}$ are complex for $(\eta-\mathrm{B})(\eta-2 \mathrm{~B})>0$,

and $\sigma_{\mathrm{r}}>0$ for $\mathrm{P}(\eta, \mathrm{B})<0$,

$$
\sigma_{\mathrm{r}}<0 \text { for } \mathrm{P}(\eta, \mathrm{B})>0,
$$

where $\mathrm{P}(\eta, \mathrm{B})=4 \mathrm{r}^{2}+(\eta-\mathrm{B})(\eta-3 \mathrm{~B} / 2)$. Because the hyperbola $\mathrm{P}(\eta, \mathrm{B})=0$ lies in the real region $(\eta-\mathrm{B})(\eta-2 \mathrm{~B})<0$, there can not exist any Hopf bifurcation, and the bifurcation diagram is qualitatively the same as Pedlosky (1981)'s including the stability (see Fig.2).

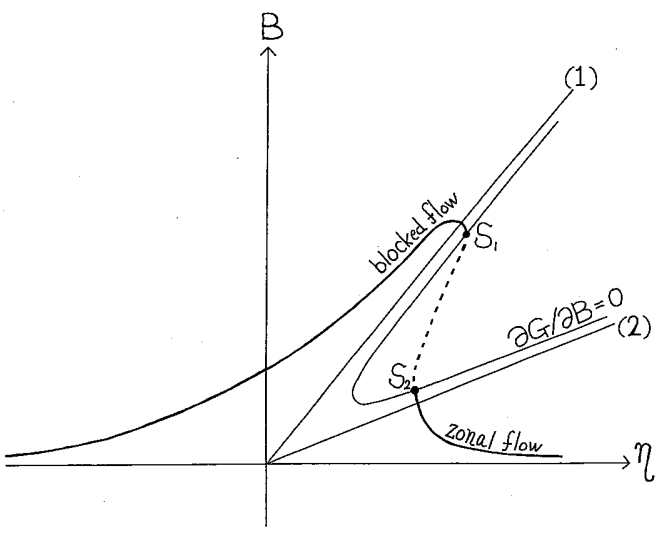

Fig. 2 Bifurcation diagram in the case of $\mathrm{D}_{\mathrm{ij}}(\Delta)=\mathrm{Q}_{\mathrm{ij}}(\Delta)(\mathrm{a}$ case of $\mathrm{b}<0)$. (1) $\eta=$ $\mathrm{B}$ and (2) $\eta=3 \mathrm{~B}$. The solid and dashed lines represent stable and unstable solutions, respectively. $\mathrm{S}_{1}$ and $\mathrm{S}_{2}$ indicate steady solution bifurcation points. 


\section{8-2. Case of dissipation proportional to Laplacian of the streamfunction}

In the remainder of this section, we consider a case of $\mathrm{D}_{\mathrm{ij}}(\Delta)=\Delta \delta_{\mathrm{ij}}$. From Eqs. $(6-4)$, (7-2) and (7-3), $\mathrm{S}_{\mathrm{i}}, \mathrm{N} / \mathrm{b}$ and $\mathrm{M}$ become

$$
\begin{aligned}
\mathrm{S}_{\mathrm{i}}= & \left(\mathrm{a}^{2} / 4 \mathrm{l}^{2}\right) \mathrm{l}_{\mathrm{i}} \mathrm{r}_{\mathrm{i}}, \\
\mathrm{N} / \mathrm{b}= & \left(4 \mathrm{l}^{2} / \mathrm{a}^{2}\right) \Sigma_{\mathrm{i}} \Sigma_{\mathrm{j}} \Sigma_{\mathrm{k}}\left(\mathrm{r}_{\mathrm{i}}{ }^{2} / \mathrm{U}_{\mathrm{i}}\right) \mathrm{Q}_{\mathrm{ij}}\left(-4 \mathrm{l}^{2}\right)^{-1} \mathrm{l}_{\mathrm{j}} \mathrm{Q}_{\mathrm{jk}}\left(-\mathrm{a}^{2}\right) \mathrm{r}_{\mathrm{k}} \\
& / \Sigma_{\mathrm{i}}\left(\mathrm{r}_{\mathrm{i}}{ }^{2} / \mathrm{u}_{\mathrm{i}}\right)^{2}, \\
\mathrm{M}= & \mathrm{Q}\left(4 \mathrm{l}^{2} / \mathrm{a}^{2}\right) \Sigma_{\mathrm{i}} \Sigma_{\mathrm{j}}\left(\mathrm{r}_{\mathrm{i}}{ }^{2} / \mathrm{U}_{\mathrm{i}}\right) \mathrm{Q}_{\mathrm{ij}}\left(-41^{2}\right)^{-1}\left(\mathrm{r}_{\mathrm{j}}{ }^{2} / \mathrm{u}_{\mathrm{j}}\right) \\
& / \Sigma_{\mathrm{i}}\left(\mathrm{r}_{\mathrm{i}}^{2} / \mathrm{u}_{\mathrm{i}}\right)^{2} .
\end{aligned}
$$

Further we restrict our attention to a case of $\mathrm{a}^{2}=2 \mathrm{~F}$. Then we can see from Eqs. (4-4) and (4-5) that the eigenvectors $r_{i}$ and $l_{i}$ become

$$
\mathrm{r}_{\mathrm{i}} \propto\left[\mathrm{u}_{1}-\mathrm{u}_{2}\right] \text { and } \mathrm{l}_{\mathrm{i}} \propto\left[\begin{array}{ll}
1 & -1
\end{array}\right] \text {, }
$$

and Eqs. (8-1) become

$$
\begin{aligned}
& \mathrm{M}=-81^{2} \Sigma_{\mathrm{i}} \Sigma_{\mathrm{j}} \mathrm{u}_{\mathrm{i}} \mathrm{Q}_{\mathrm{ij}}\left(-41^{2}\right)^{-1} \mathrm{u}_{\mathrm{j}} / \Sigma_{\mathrm{i}} \mathrm{u}_{\mathrm{i}}{ }^{2}, \\
& \mathrm{~N} / \mathrm{b}=\mathrm{M}-\delta .
\end{aligned}
$$

Here we write

$$
\left\{\left(2 \mathrm{l}^{2}\right) /\left(4 \mathrm{l}^{2}+2 \mathrm{~F}\right)\right\}\left\{\left(\mathrm{u}_{1}-\mathrm{u}_{2}\right)^{2} / \Sigma_{\mathrm{i}} \mathrm{u}_{\mathrm{i}}{ }^{2}\right\}=\delta>0 .
$$

As is easily seen from Eq. (8-3), M is bounded as

$$
2>\mathrm{M}>8 \mathrm{l}^{2} /\left(4 \mathrm{l}^{2}+2 \mathrm{~F}\right) \text {. }
$$

When $\mathrm{M}$ is minimum, $(\delta)$ has a maximum value

$$
\delta=\delta_{\max }=41^{2} /\left(41^{2}+2 \mathrm{~F}\right)<1 .
$$

For these values of $\mathrm{M}$ and $\delta$,

$$
1-\mathrm{M}+(2+\mathrm{M})(\mathrm{N} / 2 \mathrm{~b})=\delta^{2}-\delta+1<1 .
$$

Moreover, for these values of $\mathrm{M}$ and $\delta$,

$$
\mathrm{N} / \mathrm{b}=\mathrm{M}-\delta=4 \mathrm{l}^{2} /\left(4 \mathrm{l}^{2}+2 \mathrm{~F}\right)>0 .
$$

For sufficiently small r, Eqs. (7-11) and (8-7) imply that the steady solution curve corresponding to blocked flows belongs to the complex eigenvalue region. Consequently, Eqs. (7-19) and Eq. (8-6) imply that these complex eigenvalues become purely imaginary at 2 points on this steady solution curve, i.e., 2 Hopf bifurcation points emerge and the steady solutions between them lose their stability (see Fig.3).

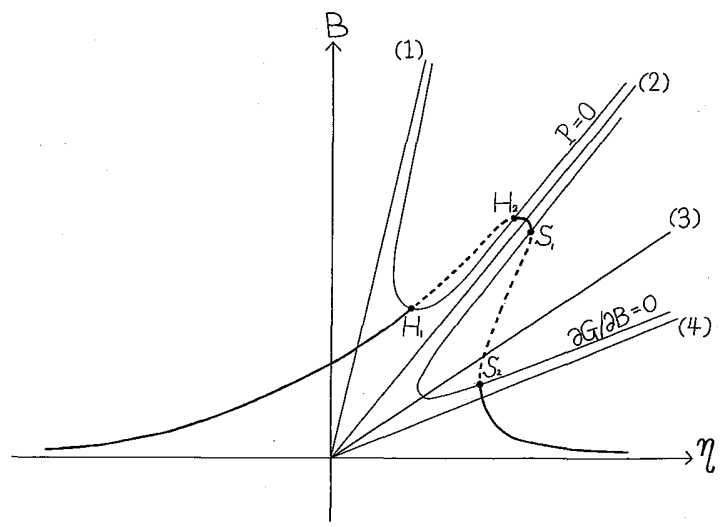

Fig. 3 Hopf bifurcations in the case of $D_{i j}(\Delta)=$ $\delta_{\mathrm{ij}} \Delta$. (a case of $\left.\mathrm{b}<0\right)$. (1) $\eta=\{1-\mathrm{M}+$ $(2+\mathrm{M})(\mathrm{N} / 2 \mathrm{~b})\},(2) \eta=\mathrm{B},(3) \eta=\{1+$ $(\mathrm{N} / \mathrm{b})\} \mathrm{B}$ and $(4) \eta=3 \mathrm{~B}$. The solid and dashed lines represent stable and unstable solutions, respectively. $S_{1}$ and $S_{2}$ indicate steady solution bifurcation points. $\mathrm{H}_{1}$ and $\mathrm{H}_{2}$ indicate Hopf bifurcation points.

\section{Conclusion}

In the above sections, we examined the dependence of the topographically forced flows in a 2-layer model on the form of dissipation. As Pedlosky (1981) showed, the bifurcation diagram is dependent on the magnitude of the dissipation, i.e., the steady solution bifurcations occur only when the coefficient of dissipation is less than a critical value $r=r_{c}$. In this note the bifurcation diagram was shown to be dependent also on the form of dissipation.

If the dissipation is taken to be proportional to the potential vorticity, then the wave-wave interaction terms vanish. As a result, there exists no Hopf bifuraction, and the diagram becomes qualitatively the same as Pedlosky (1981)'s in which the dissipation is a sum of Ekman dampings on the upper and lower boundaries and an interfacial friction between the upper and lower layers ; whereas, if the dissipation is taken to be proportional to the Laplacian of the streamfunction, i.e., Ekman dampings on the upper and lower bound- 
aries, then the wave-wave interaction terms survive, in general. As a result, 2 Hopf bifuraction points may emerge in a sub-space to which the steady solutions belong, and the steady solutions between them lose their stability. Thus, how to parameterize the dissipation due to smaller scale waves is a cru-

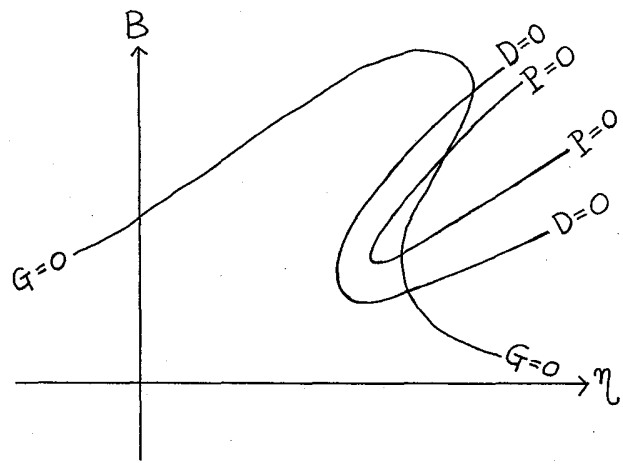

Fig. 4a The magnitude of the wave-wave interaction is not appropriate. Since the hyperbola $\mathrm{P}(\eta, \mathrm{B})=0$ on which the eigenvalues, if complex, become purely imaginary lies in the real region $\mathrm{D}(\eta, \mathrm{B})$ $<0$, there can not exist any Hopf bifurcation. $G(\eta, B)=0$ is the steady solution curve.

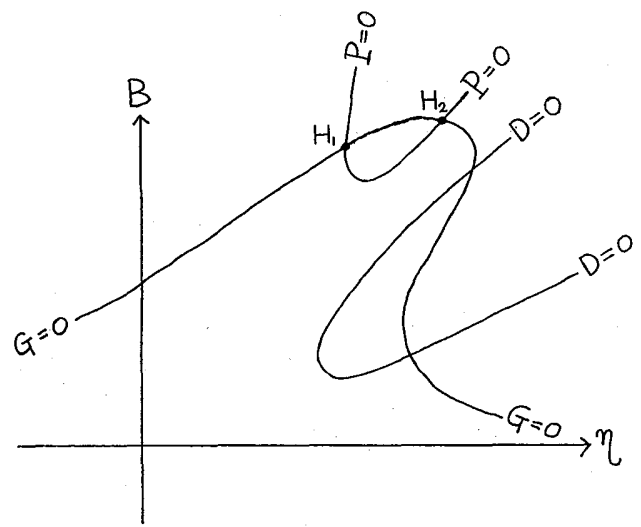

Fig. 4b The magnitude of the wave-wave interaction is appropriate. Since the hyperbola $\mathrm{P}(\eta, \mathrm{B})=0$ lies in the complex region $\mathrm{D}(\eta, \mathrm{B})>0$, there may exist 2 Hopf bifurcation points denoted by $\mathrm{H}_{1}$ and $\mathrm{H}_{2}$. cial problem.

In the case of Ekman dampings, Hopf bifurcations occur for appropriately strong wave-wave interaction and sufficiently small dissipation coefficient. The wave-wave in teraction must be appropriately strong so that the curve on which the eigenvalues, if com

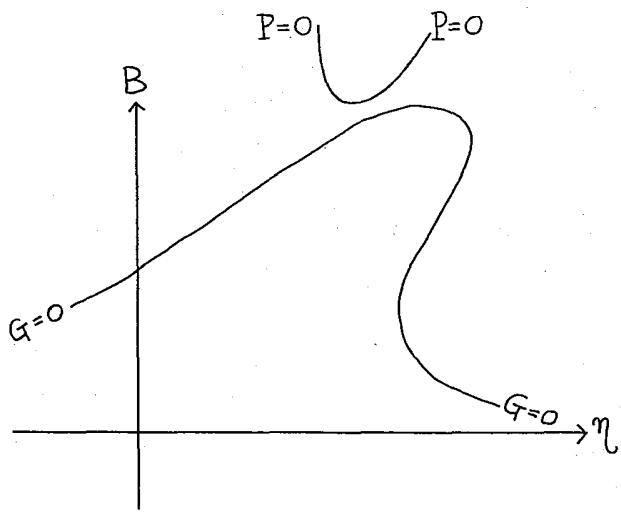

Fig. 5a. The dissipation coefficient is large enough. Since the hyperbola $\mathrm{P}(\eta, \mathrm{B})=$ 0 on which the eigenvalues become purely imaginary does not intersect the steady solution curve $\mathrm{G}(\eta, \mathrm{B})=0$, there does not exist any Hopf bifurcation.

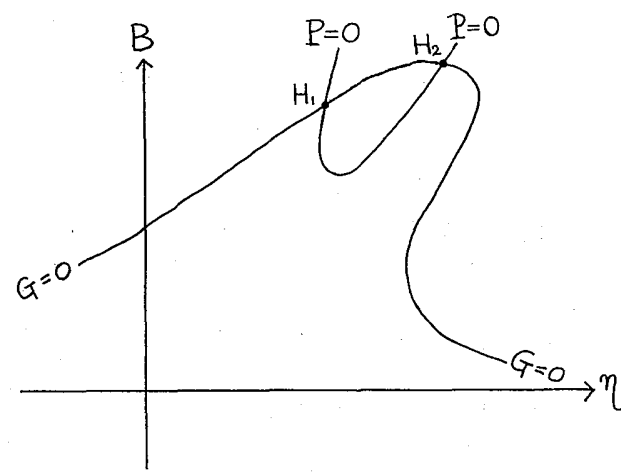

Fig. 5b The dissipation coefficient is small enough. Since the hyperbola $\mathrm{P}(\eta, \mathrm{B})=$ 0 intersects the curve $\mathrm{G}(\eta, \mathrm{B})=0$, there exist 2 Hopf bifurcation points denoted by $\mathrm{H}_{1}$ and $\mathrm{H}_{2}$. 
plex, become purely imaginary can shift into the complex-eigenvalue region (see Fig.4). As shown in section $8-2$, this indeed does occur.

The dissipation coefficient must be so small that the above curve does indeed intersect the steady solution curve (see Fig.5).

The magnitude of dissipation due to smaller scale waves has some ambiguity, and is usually assumed to be sufficiently small in the large scale dynamics. So, in this note, we also assume so small a value of dissipation that the above intersection occurs. From the equations in sections 7 and 8 , we can show that this intersection indeed occurs for a dissipation coefficient one order less than $r_{c}$, whose value is moteorologically meaningful (see Pedlosky 1981).

As was shown in the above sections, the dissipation proportional to the potential vorticity has qualitatively the same effect as Ekman dampings plus an interfacial friction. This is compatible with Klein and Pedlosky (1992)'s study for the nonlinear evolution of baroclinic waves. Moreover, the result that the dynamics with the dissipation proportional to the potential vorticity becomes simpler than with Ekman dampings is also shared with Klein and Pedlosky (1992).

It was not yet been clarified whether these are merely mathematical problems or have deep physical meanings. This belongs to our future study.

\section{References}

Klein, P. and J. Pedlosky, 1992 : The role of dissipation mechanisms in the nonlinear dynamics of unstable baroclinic waves. J. Atmos. Sci., 49, 29-48.

Pedlosky, J., 1970 : Finite amplitude baroclinic waves. J. Atmos. Sci., 27, 15-30.

Pedlosky, J., 1971 : Finite amplitude baroclinic waves with small dissipation. J. Atmos. Sci., 28, 587-597.

Pedlosky, J., 1972 : Limit cycles and unstable baroclinic waves. J. Atmos. Sci., 29, 53-63.

Pedlosky, J., 1981 : Resonant topographic waves in barotropic and baroclinic flows. J. Atmos. Sci., 38, 2626-2641.

Pedlosky, J. and C. Frenzen, 1980 : Chaotic and periodic behavior of finite amplitude baroclinic waves. J. Atmos. Sci., 37, 1177-1196.

Phillips, N. A., 1954 : Energy transformations and meridional cirulations associated with simple baroclinic waves in a two-level quasigeostrophic model.

\title{
地形に依って励起された流れの散逸形式への依存性に就いて
}

\author{
金久博忠
}

準地衡風近似の二層模型で，地形に依って励起された流れの散冕形式への依存性を解析的に考察した。特に(1) 散逸が渦位に比例する場合と(2)散逸が流線関数のラプラシアンに比例する場合即ちエクマン散逸の場合を調べ た。(1)の場合には分岐図は，エクマン散逸と上下層の界面摩擦を考慮したPedlosky (1981) と定性的に同じもの と成る。即ちブロッキング流および帯状流に対応する二つの安定定常解が同じ基本帯状流の值に対して同時に存 在する。(2)の場合には波波相互作用項が出現し，その結果分岐図に二つの周期解分岐点が生まれ，此の分岐点の 間の安定定常解が不安定化する可能性が出て来る。 